\title{
Definitive CAD/CAM-Guided Prosthesis for Immediate Loading of Bone-Grafted Maxilla: A Case Report
}

\author{
Herman K. Kupeyan, BSc., DDS, MS;* Matt Shaffner, BSc., DDS; ${ }^{\dagger}$ \\ Joseph Armstrong, BSc., DDS, MSc., FRCD $(C)^{\ddagger}$
}

\begin{abstract}
Background: The reconstruction of a functional occlusion in the presence of severe residual ridge resorption remains a significant challenge for maxillofacial surgeons and prosthodontists. Removable appliances are unsuccessful in severely resorbed cases and the advanced degree of bone loss precludes conventional implant-retained prostehesis. In recent years, bone graft reconstruction of the maxilla and the mandible has been advocated to reverse the debilitating effects of alveolar ridge resorption.
\end{abstract}

Purpose: The surgical objective was the reconstruction of the osseous platform of the jaws, thereby allowing implantretained definitive prosthetic rehabilitation utilizing the Teeth-in-an-Hour ${ }^{\mathrm{TM}}$ concept within 4 months.

Materials and Methods: A completely edentulous patient received bone grafts from the posterior iliac crest to augment both the maxillary and mandibular alveolar ridges. The manmandible was augmented using Marx's "soft issue matrix expansion procedure" and was restored in the conventional manor using an impression and a milled framework. The maxilla underwent "inlay/onlay" bone graft augmentation of the right and left maxilla at the same time as the mandiblular procedure. Implant placement was at 4 months post-bone graft reconstruction. Immediate loading of implants into a bone graft using Nobel Biocare's Teeth-in-an-Hour ${ }^{\mathrm{TM}}$ protocol prelaunch was implemented. This included CT-based implant planning, simultaneous CAD/CAM fabrication of a surgical guide, a CNC-milled framework, flapless surgical placement of the implants, and immediate placement of a pre-fabricated definitive prosthesis. This approach reduced the time necessary for an edentulous patient to transform from severely atrophic alveolar support to implant-retained maxillary and mandibular prosthetic restorations. Immediate loading with an implant retained definitive prosthesis using a bone graft-reconstructed osseous platform was also performed.

Conclusion: The Teeth-in-an-Hour ${ }^{\mathrm{TM}}$ concept was utilized to restore bone grafted augmentation of the maxilla with immediate loading of a definitive prosthesis within 4 months. The remarkable accuracy of the software, minimally invasive and short surgery and treatment time, uneventful quick recovery with minimal discomfort is a benefit not only to the patient, also to the treating team.

KEY WORDS: Teeth-in-an-Hour, definitive CAD/CAM prosthesis, grafted maxilla, implant placement

\footnotetext{
*Private practice, Windsor, Ontario, Canada; adjunct associate professor, University of Michigan, Ann Arbor, MI, USA; ${ }^{\dagger}$ oral and maxillofacial surgery intern, London Health Sciences Center and University of Western Ontario, London, Ontario, Canada; "oral and maxillofacial surgeon and chief of dentistry, London Health Sciences Center; adjunct clinical professor, Oral and Maxillofacial Surgery Schulich School of Medicine and Dentistry, University of Western Ontario, London, Ontario, Canada
}

Reprint requests: Herman K. Kupeyan, BSc., DDS, MS, 1100 Ouellette Avenue, Windsor, Ontario, Canada N9A 1C9; e-mail: dr.k@smileandbody.com

(C) 2006 Blackwell Publishing, Inc.

DOI 10.1111/j.1708-8208.2006.00018.x
The advent of modern endosseous implant design and improved surface technology has allowed the development of new restorative techniques that decrease a patient's total treatment time. The original restoration of titanium implants based on the Branemark protocol required 3 to 6 months for osseointegration prior to restoration, depending on implant position and bone quality. ${ }^{1,2}$ Successful immediate and early loading of implants has been described in multiple studies using the latest generation fixtures. ${ }^{3-5}$ Rigid cross-arch stabilization has been shown to successfully allow the immediate loading of titanium implants in an edentulous 
ridge, allowing rapid sequence rehabilitation of the arch involved. With the combination of the new surface technology and cross-arch stabilization, immediate placement of an implant-supported fixed prosthesis following implant placement is now a predictable treatment option. ${ }^{3,6-10}$

There has been numerous treatment modalities described for the immediate loading of implants during the rehabilitation of the edentulous ridge. Laboratoryfabricated transitional prosthesis can be processed following the placement of the implants by making a traditional impression of the fixtures. Retrofitting an existing complete denture by providing relief in the areas of the implants and using acrylic to create a retentive framework is known as conversion prosthesis. ${ }^{11}$ The fabrication of the temporary prosthesis following the surgical stage of implant placement can be difficult for the patient and costly to the restorative dentist because of the extended time in the chair.

The following case report describes the rehabilitation of a completely edentulous patient who has received bone grafts from the posterior iliac crest to augment both the maxillary and mandibular alveolar ridges. The mandible was augmented using Marx's "soft tissue matrix expansion procedure" and was restored in the conventional manner using an impression and a milled framework. ${ }^{12}$ The maxilla underwent "inlay/onlay" bone graft augmentation of the right and left maxilla at the same time as the mandibular procedure. Implant placement was at 4 months post-bone graft reconstruction. There has not been, to our knowledge, a documentation in the literature of immediate loading of implants with a final prosthesis into a bone-grafted reconstruction of the jaw. This report discusses a technique for immediate loading of implants into a bone graft using Nobel Biocare's Teeth-in-an-Hour ${ }^{\mathrm{TM}}$ (Nobel Biocare Göteborg, Sweden) protocol prelaunch. This includes CT-based implant planning, simultaneous CAD/CAM fabrication of a surgical stent, a CNC-milled framework, flapless surgical placement of the implants, and immediate placement of a pre-fabricated definitive prosthesis. This systematic approach to full mouth rehabilitation reduces the time necessary for an edentulous patient to go from severely atrophic alveolar support to implantretained maxillary and mandibular prosthetic restoration. This report also documents the immediate loading with an implant retained definitive prosthesis using a bone graft-reconstructed osseous platform.

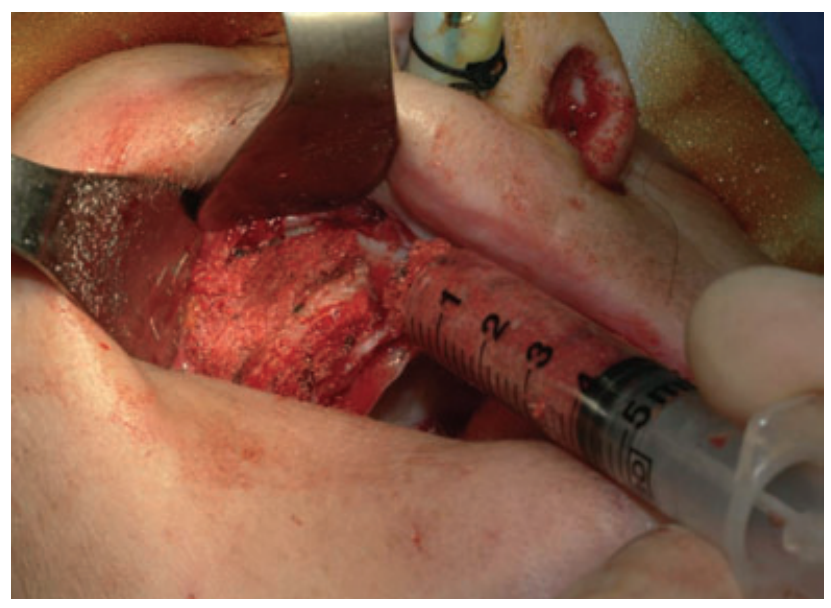

Figure 1 Packing of morselized corticocancellous bone around the fixed cortical blocks.

\section{CASE REPORT}

A 68-year-old woman presented with a maxillary complete denture and an IMZ ${ }^{\circledR}$ implant-supported mandibular complete denture (Friedrichsfeld AG, Mannheim, Germany). The patient had been edentulous since she was 13 years old. Because of over 50 years of removable complete denture use, the edentulous alveolar ridges have suffered severe resorption. The patient complained of ill-fitting maxillary and mandibular prosthesis and demonstrated over-closure leading to a pseudo class III occlusion. The patient stated that she was interested in fixed prosthetic rehabilitation. After a complete examination by a prosthodontist and oral surgeon, it was determined that the patient would require maxillary and mandibular alveolar ridge augmentation and bilateral sinus lifts in order to allow sufficient bone volume for the placement of endosseous implants (Figure 1).

Following sufficient time for consolidation of the bone grafts, the second prosthetic stage of the sequence began. The patient was reevaluated to determine tooth position for aesthetics, phonetics, and appropriate vertical dimension, by fabricating transitional upper and lower dentures.

Once the appropriate setup was achieved the mandibular arch rehabilitation started by uncovering the mandibular implants that were placed during the tent pole procedure. A full thickness flap was raised by making an incision along the alveolar crest exposing the grafted bone. Excess bone was removed from around the implants and the cover screws were detached. Healing abutments were placed onto the implants and the inci- 
sion was closed. A conventional protocol was followed during the reconstruction of the mandibular arch. After 2 to 3 weeks of healing post-second-stage implant exposure, an impression using a reversible hydrocolloid was made for a study cast poured in a gypsum stone. This study cast was used to fabricate an open-top custom tray from a light-cured resin. At a subsequent appointment, healing abutments were removed individually and replaced by impression copings (Nobel Biocare 29073). If the impression copings seating could not be confirmed by visual inspection, a radiograph was taken to verify the seating prior to impression taking. The impression copings were luted together utilizing a resin material in the areas of long span. The resin was sectioned and reluted to compensate for any dimensional change of the resin material. A PVS medium body impression is made for the fabrication of the master cast. Laboratory analogs (Nobel Biocare 29108) were attached to the impression copings, and a soft tissue and stone master cast was fabricated.

The interim management post bone augmentation surgery would involve modifying the existing prosthesis as needed. The necessary tissue conditioning (Viscogel ${ }^{\circledR}$, Densply, York, PA, USA) and relines were provided for the patient as the healing process continued. The transitional prosthesis was used as a guide for the fabrication of the fixed mandibular acrylic provisional and the occlusion was further developed with the interim removable maxillary prosthesis.

Once the mandibular provisional was acceptable both functionally and aesthetically, it was then used as a guide for the maxillary prosthesis. If the fabricated transitional maxillary prosthesis is acceptable to the patient aesthetically, the practitioner needs to confirm its stability or a reline would be required. It is imperative at this stage that both the patient and practitioner are satisfied prior to proceeding with the projected immediately loaded fixed prosthesis. This commits both the patient and practitioner to the final restoration; otherwise a new setup should be undertaken. In this procedure, the transitional denture is duplicated into clear acrylic for use as a radiographic guide. Six radiographic markers are placed randomly on the labial aspect. These holes of $1.5 \mathrm{~mm}$ are filled with gutta-percha in a spherical form (Figure 2A). As with all duplications, confirming the seat of the duplicate radiographic guide is imperative. Once the existing prosthesis and the duplicate radiographic guide were ideal and identical in aes-
A

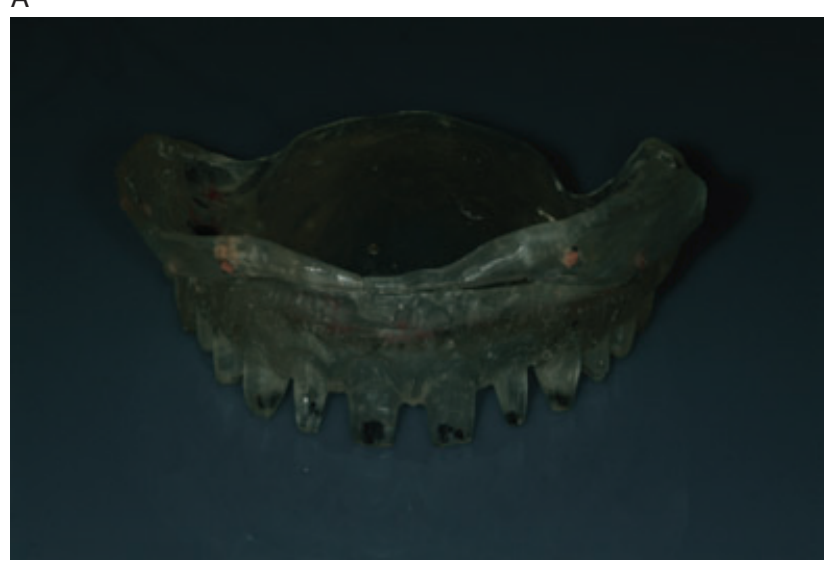

B

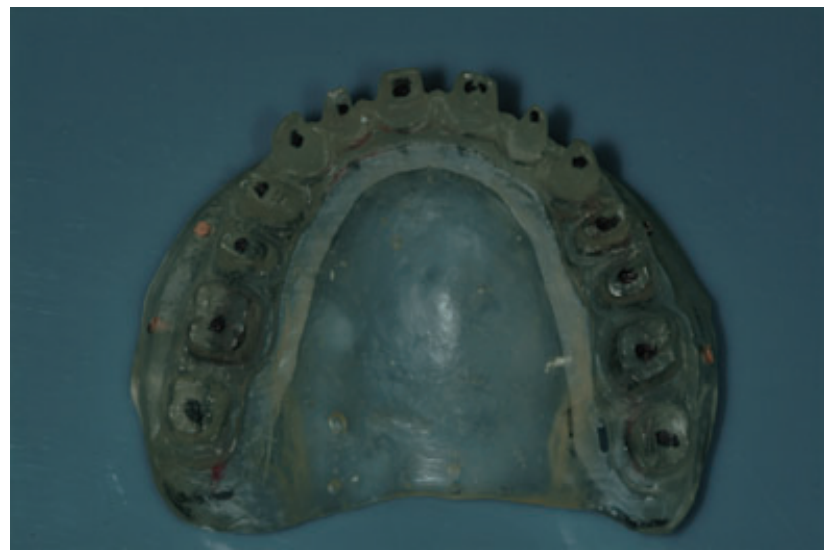

Figure 2 Modified duplicate of transitional prosthesis with radiographic markers ready for $\mathrm{CT}$ scan (A). Tooth preparation on the radiographic guide (B).

thetics and fit, the radiographic guide was prepared to facilitate the fabrication of the Procera ${ }^{\circledR}$ Implant Bridge (Nobel Biocare AB, Göteborg, Sweden) and individual crowns (Figure 2B). An interocclusal record was made with a rigid vinyl polysiloxane at the patient's appropriate centric position and occlusal vertical dimension. At this point a CT scan of the maxilla was generated using a two-scan technique. ${ }^{13}$ The first scan was of the patient with the interim denture in situ, which included the radiopaque markers and the interocclusal record. The second scan was of the denture only. The CT data was converted into a file format compatible with the planning program, having the two sets of scans superimposed upon each other. One scan was of the osseous tissues and the other of the complete denture, which showed the position of the planned denture teeth in relation to the bone. The reformatted data was returned for planning of the implant positions. The threedimensional Procera ${ }^{\circledR}$ Software Planning program (Nobel Biocare $\mathrm{AB}$ ) allowed the clinicians to evaluate the 


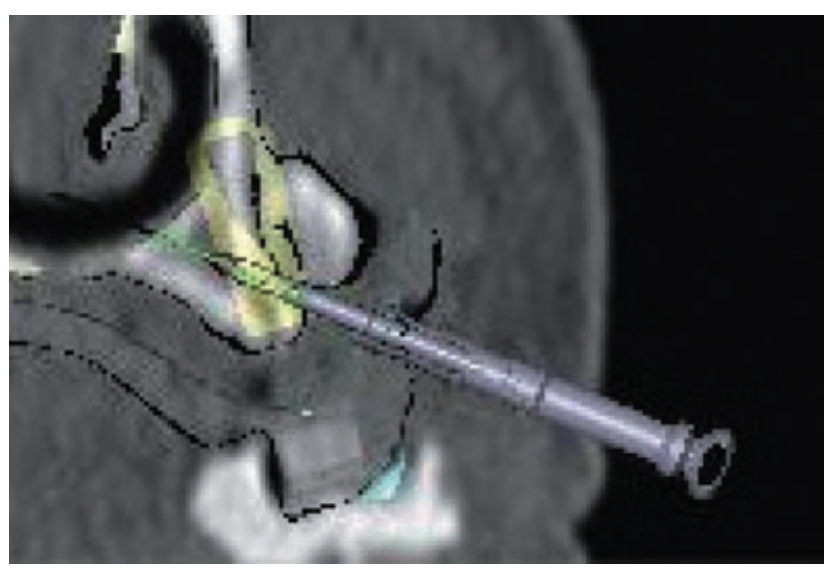

Figure 3 Virtually placed implant and anchor pin in twodimensional view.

osseous tissues in relation to the position of the denture teeth. The tooth position on the radiographic guide is viewed in a two-dimensional window. The implant and the anchor pin are installed. The augmented ridge width is clearly visible on the labial aspect (Figure 3). The augmented bone is seen labially (Figure 4). The yellow zone seen surrounding the implants denotes a safety zone of $1 \mathrm{~mm}$. The implants are positioned within host and grafted bone (Figure 5). The sizes and positions of the dental implants were selected digitally and virtually placed utilizing the radiographic guide ${ }^{14}$ (Figure 6). The surgical placement data was transferred to a milling center for fabrication of the stereolithography cast and the surgical guide to provide the exact positions of each dental implant during surgery. ${ }^{15}$

\section{Dental Laboratory}

Implant replicas were placed in the preplanned osteotomy sites, providing the exact positions of the future implants. The stereolithography cast was delivered to the dental laboratory. It was mounted to the opposing cast with the original interocclusal record and

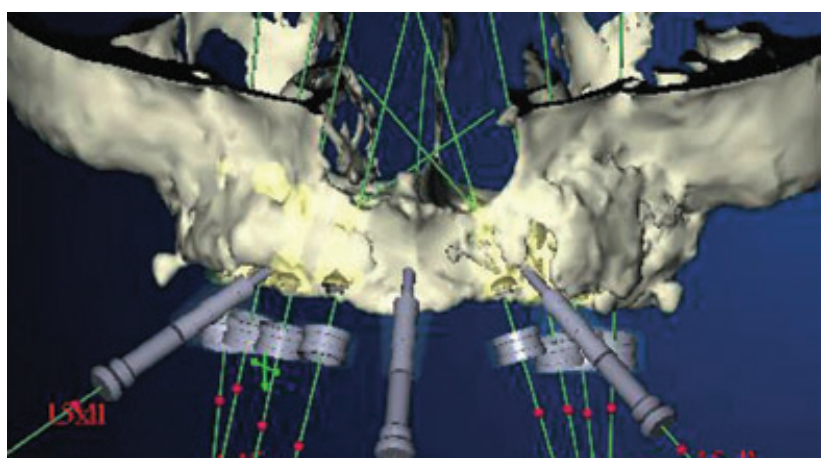

Figure 4 Labial view showing implants surrounded by grafted bone and safety zone of surrounding bone (yellow).

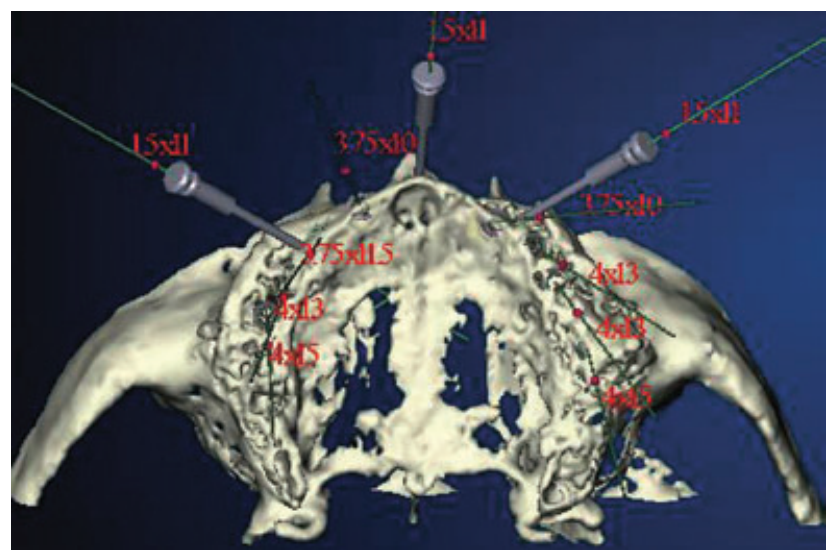

Figure 5 Position of implants in grafted bone.

radiographic guide on an articulator. A new interocclusal record was made between the surgical template and the opposing arch on the articulator to facilitate positioning and to transfer the centric relation and occlussal vertical dimension information to the patient during surgery (Figure 7). At the time of surgery the surgical template is secured to the osseous structure of the maxilla by horizontal pins. The surgical template therefore provides the capability to transfer the exact implant positions to the patient. The definitive prosthesis was fabricated on the stereolithography cast prior to implant placement. The esthetics and phonetics of the definitive prosthesis was dictated by the interim denture. The prosthesis was fabricated from milled titanium using the Procera Implant Bridge protocol (Figure 8). ${ }^{13}$ The titanium

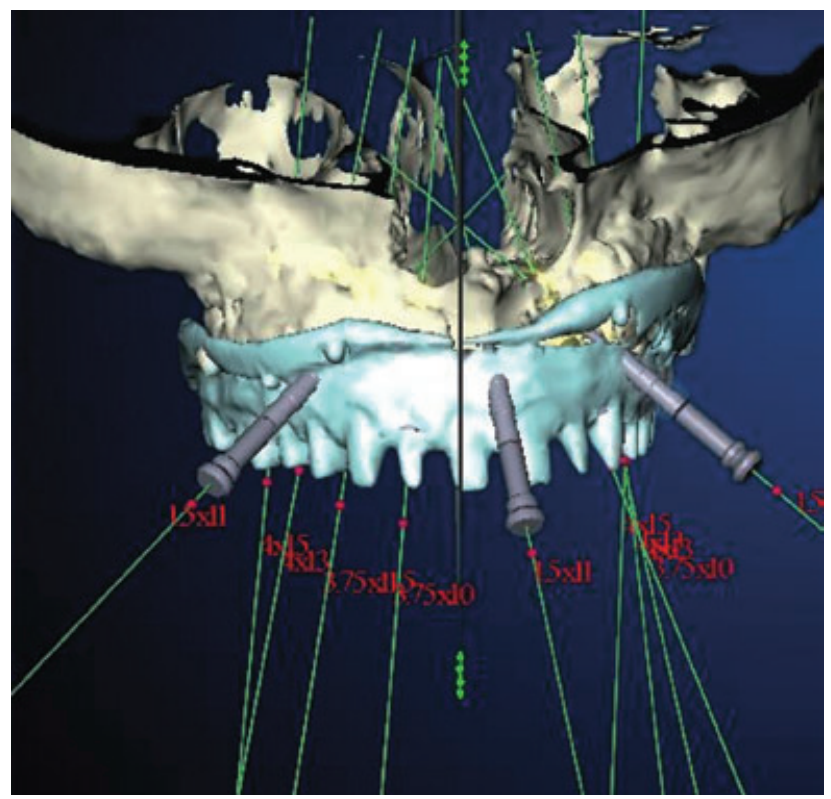

Figure 6 Procera ${ }^{\circledR}$ Software Planning program allows for threedimensional views for implant planning. Completed surgical plan with eight implants and three horizontal stabilization pins. 


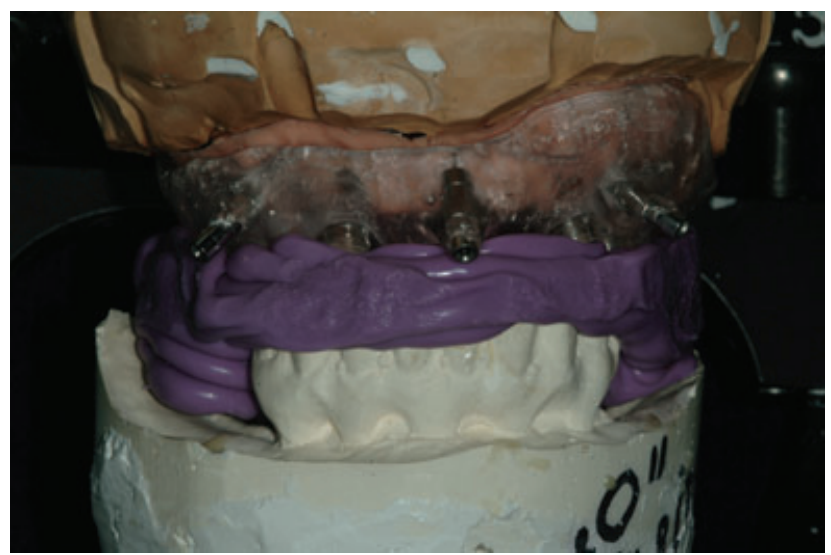

Figure 7 Stereolithography cast mounted on an articulator, surgical template, and interocclusal record used during the surgery.

framework was prepared for porcelain application. A compatible porcelain (Triceram opaque porcelain, EspriDent, Inspringen, Germany) was applied to gingival segments and the neutral opaque to the tooth portions. An impression was taken with a PVS material (Double Take, Ivoclar Vivadent AG, Schaan, Liechtenstein), and individual dies were fabricated on a new master cast in the usual manner utilizing a gypsum stone (Silky Rock, WhipMix, Louisville, KY, USA). The dies were scanned according to the Procera protocol. Once the aluminous oxide copings were obtained a veneering porcelain was applied (Ducera Allceram, DeguDent GmbH, Hanau/Wolfgang, Germany) (Figure 9).

\section{Surgical Procedure}

The surgical procedure was performed according to the Teeth-in-an-Hour concept (Nobel Biocare AB). The flapless surgery was performed under local anesthetic. ${ }^{16,17}$ The surgical template was inserted and posi-

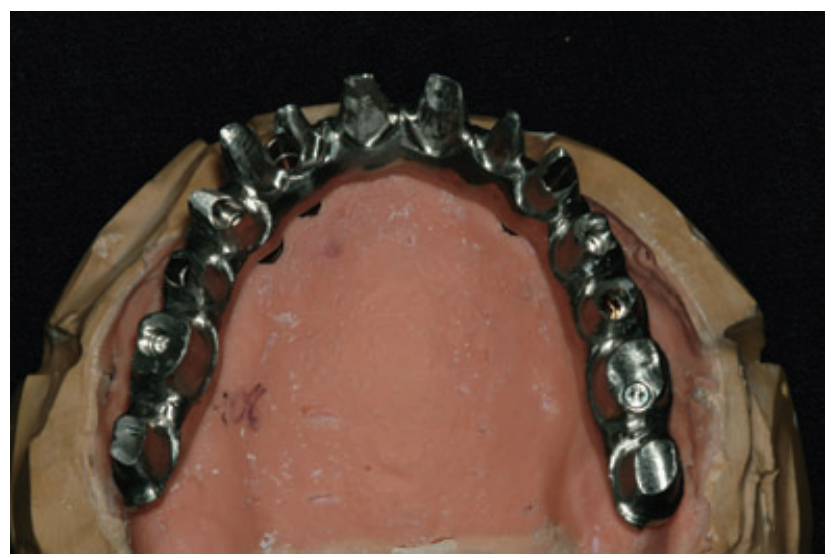

Figure 8 Milled Procera ${ }^{\circledR}$ Implant Bridge returned for porcelain application.

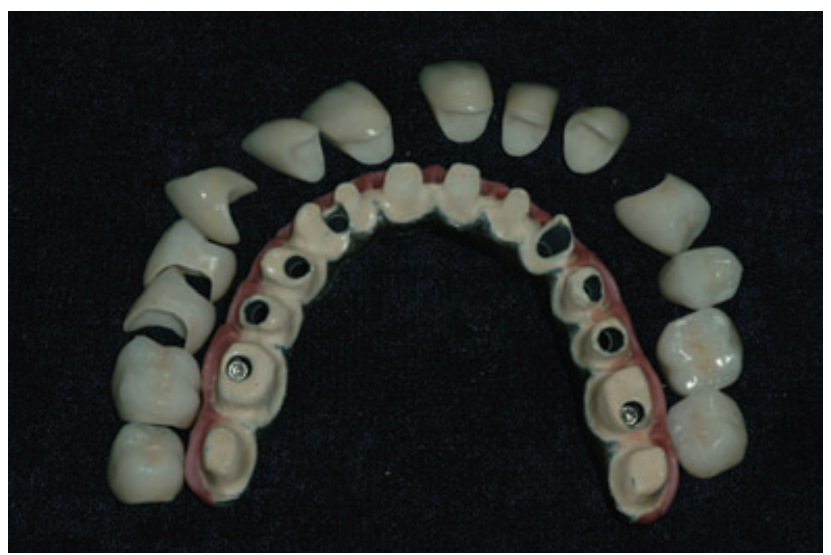

Figure 9 Completed Procera ${ }^{\circledR}$ Implant Bridge and individual crowns.

tioned with the centric relation interocclusal record. While the patient was closed into the interocclusal record, the initial step was to place the stabilization pins in the three preplanned horizontal positions while the patient closed her mouth into the interocclusal record. The 1.5-mm twist drill was used to create retentive holes for the stabilization pins. The pins were positioned, securing the surgical template, and the patient was allowed to open her mouth. The first osteotomy site was prepared, adjacent to the most anterior implant site, using the appropriate drilling guides and twist drills. The preplanned length and diameter of the implant was placed using the implant guide. After insertion of the implant (Brånemark System ${ }^{\circledR}$, Nobel Biocare $\mathrm{AB}$ ), a template abutment was inserted, which connected the surgical template to the placed implant, providing additional stability to the template.

The next implant was inserted in the opposite side of the arch, also adjacent to the most anterior implant. After the implant was placed, a second template abutment was inserted. The three horizontal stabilization pins, and the two template abutments, ensured the surgical template's stability and accurate position. The remaining implants were placed, using the same drilling guides and twist drills.

\section{Prosthetic Procedure}

After all implants were placed, the pre-fabricated definitive prosthesis framework was inserted (Figure 10). Vertically adjustable abutments (Nobel Biocare AB) were used, which allowed for discrepancies in the $\mathrm{z}$-axis. The adjustable abutment is an interlocking cylinder sleeve with two pieces that slide within one another. The 


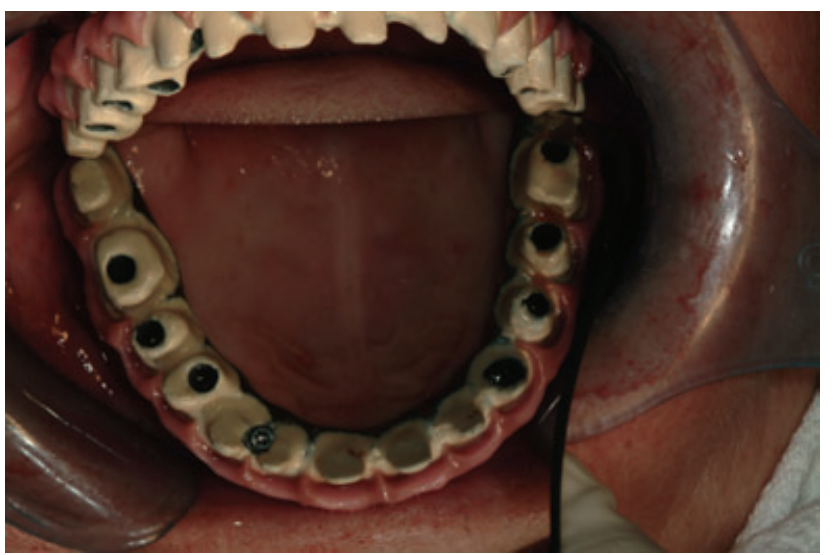

Figure 10 Procera ${ }^{\circledR}$ Implant Bridge seated prior to crown insertion.

sliding cylinders of the abutment allow for slight variations in the $\mathrm{z}$-axis position of the implant, allowing a passively fitting framework. If the patient is able, postoperative radiographs can be made to confirm the fit of the framework. During this procedure radiographs were completed upon stabilizing the framework, torquing the retaining screws at $35 \mathrm{Ncm}$, and sealing the access holes with a cotton pellet and gutta-percha. The individual aluminous oxide crowns were cemented using a resin cement (Panavia F Dual Cure Dental Adhesive, Kuraray Dental Systems, New York, NY, USA) in areas where no access holes existed, and a provisional acrylic cement was utilized to cement the crowns in the areas of the screw access holes (Improv Temp Cement, Nobel Biocare Göteborg, Sweden). The occlusion was evaluated and adjusted to have simultaneous centric relation contact and a canine-protected occlusal scheme (Figure 11). Following the procedure, the patient was discharged home and instructed to maintain a soft diet for the next 6 weeks.

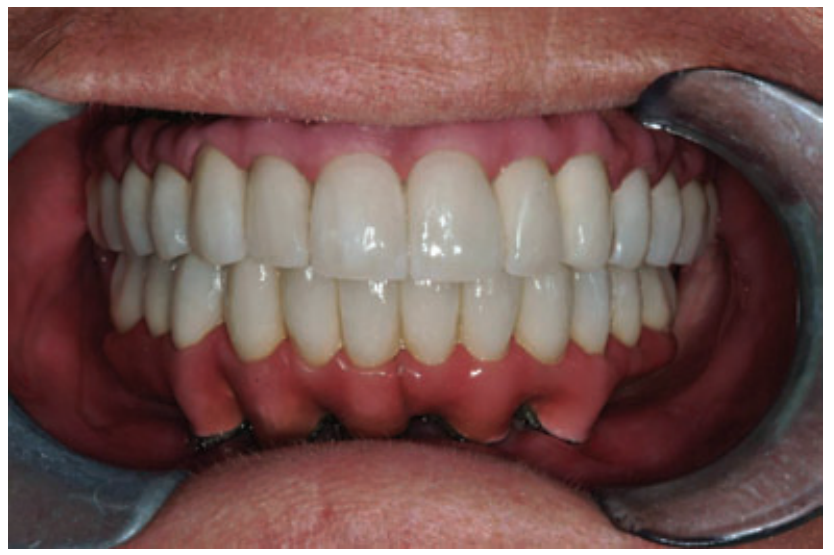

Figure 11 Interocclusal view of completed prosthesis upon insertion.

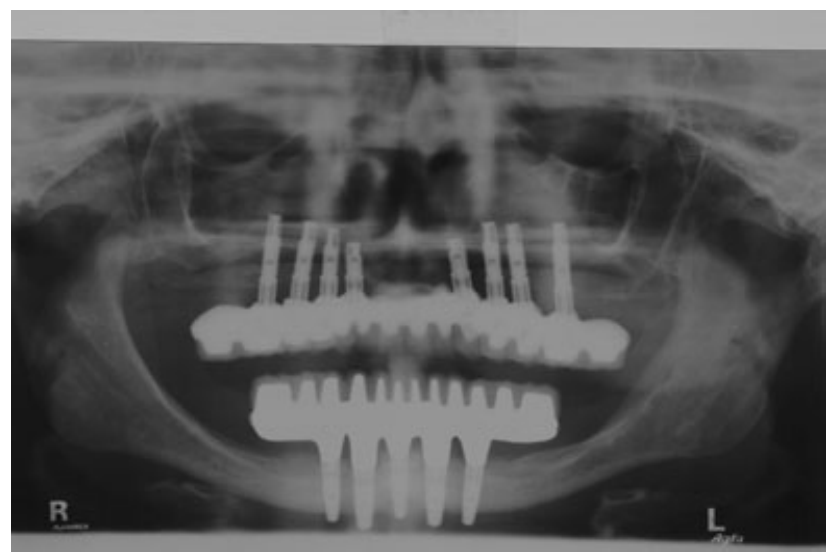

Figure 12 Final radiograph 1 week post-op.

\section{DISCUSSION}

The Teeth-in-an-Hour protocol is a unique solution made possible by the Procera System. With the aid of the CT scans and a virtual planning software, a custom fabricated precision drill guide and a premanufactured prosthesis can be made before surgery. During the clinical and digital planning stage most treatment decisions are made with regard to prosthesis design and implant placement. The execution of implant placement is performed with a flapless procedure that results in minimal surgical intervention. This results in a short and nontraumatic surgery with a minimum of postoperative complications, allowing the patient to leave the chair with a fixed prosthesis in place after only 1 hour. The patient was elated with the short surgery time and remarked postoperatively at the uneventful short recovery with minimal discomfort. No sequellae of swelling or bruising was associated with this procedure. The mapping of the maxillary topography for fabrication of implant guides is so accurate that we have used this method to restore bone graft augmentation of the maxilla with immediate loading of a definitive prosthesis. This new protocol simplifies the operative component of total arch reconstruction and allows transition from severe ridge resorption and failing conventional dentures to an implant fixed prosthesis within 4 months, which is the time required for the bone graft to consolidate (Figure 12).

\section{REFERENCES}

1. Branemark PI. Osseointegration and its experimental background. J Prosthet Dent 1983; 50:339-410. 
2. Adell R, Leckholm U, Rochier B, Branemark PI. A 15 year study of osseointegrated implants in the treatment of the edentulous jaw. Int J Oral Surg 1981; 10:387-416.

3. Gapshi R, Wang HL, Mascarenhas P, Lang NP. Critical review of immediate implant loading. Clin Oral Implants Res 2003; 14:512-527.

4. Ogawa T, Nishimura I. Different bone integration profiles of turned and acid-etched implants associated with modulated expression of extracellular matrix genes. Int J Oral Maxillofac Implants 2003; 18:200-210.

5. Zechner W, Tangi S, Furst G, et al. Osseus healing characteristics of three implant types. Clin Oral Implants Res 2003; 14:150-157.

6. Tarnow D, Emitiaz S, Classi A. Immediate loading of threaded implants at stage 1 surgery in edentulous arches: ten consecutive case reports with 1-5 year follow-up. Int J Oral Maxillofac Implants 1997; 12:319-324.

7. Chatzistavrou M, Fenton DA, Cooper F. Immediate loading of dental implants in partially edentulous patients: a clinical report. J Prosthodont 2003; 12:26-29.

8. Harris D, Buser D, Dula K, et al. EAO guidelines of the use of diagnostic imaging in implant dentistry. A consensus workshop organized by the European Association for Osseointegration in Trinity College Dublin. Clin Oral Implant Res 2002; 13:566-570.

9. Glauser R, Lundgren AK, Gottlow J, et al. Immediate occlussal loading of Branemark TiUnite implants placed predominately in soft bone: 1-year results of a prospective clinical study. Clin Implant Dent Relat Res 2003; 5(Suppl 1):4756.

10. Sadowsky SJ. The implant supported prosthesis for the edentulous arch: design considerations. J Prosthet Dent 1997; 8:28-33.
11. Zitmaqnn NU, Marinello CP. Treatment plan for restoring the edentulous maxilla with implant supported restorations: removable overdentures versus fixed partial denture design. J Prosthet Dent 1999; 82:188-196.

12. Marx RE, Schellenburger T, Wimsatt J, Correa P. Severely resorbed mandible: predictable reconstruction with soft tissue matrix expansion (tent pole) grafts. J Oral Maxillofac Surg 2002; 60:878-888.

13. van Steenberghe D, Naert I, Andersson M, Brajnovic I, Van Cleynenbreugel J, Suetens P. A custom template and definitive prosthesis allowing immediate implant loading in the maxilla: a clinical report. Int J Oral Maxillofac Implants 2002; 17:663-670.

14. Verstreken K, van Cleynenbreugel J, Marchal G, Naert I, Suetens P, Van Steenberghe D. Computer assisted planning of oral implant surgery. A three-dimensional approach. Int J Oral Maxillofac Implants 1996; 11:806-810.

15. Parel SM, Funk JJ. The use and fabrication of a selfretaining surgical guide for controlled implant placement: a technical note. Int J Oral Maxillofac Implants 1991; 6:207-210.

16. Rocci A, Martignoni M, Gottow J. Immediate loading in the maxilla using flapless surgery, implants placed in predetermined positions, and prefabricated provisional restorations: a retrospective 3-year clinical study. Clin Implant Dent Relat Res 2003; 5(Suppl 1):29-36.

17. Campelo LD, Camara JR. Flapless implant surgery: a 10 year clinical retrospective analysis. Int J Oral Maxillofac Implants 2002; 17:271-276. 